EGU21-319

https://doi.org/10.5194/egusphere-egu21-319

EGU General Assembly 2021

(c) Author(s) 2021. This work is distributed under

the Creative Commons Attribution 4.0 License.

\title{
Imaging the subsurface to inform hydrological models: a geophysicist's perspective
}

\author{
Thomas Hermans ${ }^{1}$, Hadrien Michel ${ }^{1,2,3}$, Jorge Lopez-Alvis ${ }^{1,2}$, and Frédéric Nguyen ${ }^{2}$ \\ ${ }^{1}$ Ghent University, Faculty of Sciences, Department of Geology, Ghent, Belgium (thomas.hermans@ugent.be) \\ ${ }^{2}$ Liege University, Faculty of Applied Sciences, Liege, Belgium \\ ${ }^{3}$ FRS-FNRS, Brussels, Belgium
}

Heterogeneity plays a major role in subsurface processes from the local scale (preferential infiltration and flow paths, fractures) to the catchment scale (presence of lateral and vertical variability, multiple horizons, bedrock interface, etc.). If high-resolution direct observations are often available through drillholes, CPT or installing in-situ monitoring probes, those local measurements only provide punctual or 1D information. Within this context, geophysical techniques can provide relevant spatially-distributed information (2D, 3D or even 4D) with a much larger coverage than direct measurements. However, geophysical information remains indirect and must be translated into the sought parameter through petrophysical or transfer functions.

Geophysicists are facing two important issues when imaging the subsurface: 1) Generating images of the subsurface that are consistent in terms of soil or geological structures; 2 ) Integrating the geophysical information into hydrological models. Both issues will be discussed in this contribution.

Geophysical imaging is the result of an inversion process whose solution is non-unique. This problem is generally solved using a regularization approach introducing some a priori characteristics of the model. The dominant choice is still the smoothness constraint inversion, which often introduces a too simplistic representation of the subsurface, and decreases the potential of geophysics to discriminate between different facies. In the first part of this contribution, we will analyze what can be expected from geophysical methods in terms of characterization of the heterogeneity. We will illustrate how the inversion method affects the discrimination potential of geophysics, and how we can improve the geophysical image by accounting for prior information. We will see how the discrimination potential decreases with the loss of resolution. Finally, we will investigate how recent methodologies using machine learning can improve our ability to image the subsurface.

Given the high spatial coverage of geophysical methods, they have a huge potential to inform hydrological models in terms of heterogeneity. However, the limitations related to geophysical inversion also make the geophysical model uncertain and the risk to propagate erroneous information exists. In the second part of this contribution, we will illustrate how to incorporate geophysical data into hydrological models to unravel their spatial complexity. At the early stage of 
a project, several scenarios regarding spatial heterogeneity are often possible (orientation of fractures, number of facies to consider, interconnection within one facies, etc.), and this can largely influence the outcomes of the hydrological models. In this context, geophysical data can be used to verify the consistency of some scenarios without requiring any inversion in a process called falsification. Once realistic scenarios have been identified, geophysical data can be used to spatially constrain hydrological models. However, this should ideally account for the uncertainty related to geophysical inversion. One possibility is to use a fully-coupled approach where geophysical data are integrated directly in the hydrological model inversion. This requires nevertheless a transfer function to relate hydrological and geophysical variables. As an alternative, a sequential approach using a probabilistic framework accounting for the imperfect geophysical data can be used. The latter requires co-located measurements. 